\title{
Application of (1S,4S)-2,5-diazabicyclo[2.2.1]heptane derivatives in asymmetric organocatalysis: the Biginelli reaction
}

\author{
Rodrigo González-Olvera, ${ }^{a}$ Patricia Demare, ${ }^{\mathrm{b}}$ Ignacio Regla, ${ }^{\mathrm{b}}$ and Eusebio Juaristi*a \\ ${ }^{a}$ Departamento de Química, Centro de Investigación y de Estudios Avanzados del Instituto \\ Politécnico Nacional, Apartado Postal 14-740, 07000 México, D. F., México \\ ${ }^{b}$ Facultad de Estudios Superiores Zaragoza, Universidad Nacional Autónoma de México, 09230 \\ México, D. F., México \\ E-mail: juaristi@relaq.mx
}

\section{Dedicated to Professor Torbjorn Norin in appreciation of his contributions to organic chemistry}

\begin{abstract}
This report describes the application of three chiral bicyclic diamines as organocatalysts in the enantioselective Biginelli reaction. The product in this reaction is 3,4-dihydropyrimidin-2(1H)-one (DHPM), a heterocycle of great importance owing to its interesting biological activity. It was found that $(1 S, 4 S)$-2,5-diazabicyclo[2.2.1] heptane $2 \mathrm{HBr}(\mathbf{1})$ and its $N$-methylated derivative 2 effectively catalyze the reaction between ethyl acetoacetate, representative aromatic aldehydes, and urea to afford the expected DHPMs in good yields and moderate enantioselectivities, $18-37 \%$ ee, favoring the $(S)$ enantiomer (like enantioinduction). Better yields (up to $94 \%$ ) and slightly higher enantiomeric excess (up to $46 \%$ ee) of the desired DHPMs were obtained in the presence of 10 mol\% of the hydrobromide salt $(1 S, 4 S)-2-[(R)-1$-phenylethyl]-2,5-diazabicyclo[2.2.1]heptane $2 \mathrm{HBr}$
\end{abstract} (3).

Keywords: Multicomponent reactions, Biginelli reaction, asymmetric organocatalysis, (1S,4S)-2,5diazabicyclo[2.2.1] heptane, 3,4-dihydropyrimidin-2(1H)-one

\section{Introduction}

Multicomponent reactions (MCRs) are those involving three or more reagents that are combined in a single chemical step to afford a product containing segments of the starting substrates. ${ }^{1}$ Multicomponent reactions are of great interest to synthetic organic chemists because the desired product may be obtained in a single experimental step, reducing the time and cost of the process. A salient example of MCR is the Biginelli reaction ${ }^{2 a}$ developed in 1893 by the Italian chemist Pietro Biginelli, who described the preparation of 3,4-dihydropyrimidin-2(1H)-one (DHPM) via the 
cyclocondensation of ethyl acetoacetate, benzaldehyde and urea under acidic conditions (Scheme $1)^{2}$<smiles></smiles>

DHPM

\section{Scheme 1}

It has been found that several DHPM derivatives possess biological activity, which has led to their use as antihypertensive, antiviral, antibacterial or anticancer agents. ${ }^{3}$ Furthermore, DHPMs are chiral compounds and it has been discovered that the configuration at the stereogenic carbon $\mathrm{C}(4)$ determines their biological properties. For example, only the enantiomer $(R)$-SQ 32926 exhibits an antihypertensive effect, ${ }^{4}$ and only $(S)$-Monastrol ${ }^{5}$ and $(R)$-Mon- $97^{6}$ present potential anticancer activity (Figure 1).<smiles>CC1=C(C(=O)OC(C)C)C(c2cccc([N+](=O)[O-])c2)N(C(N)=O)C(=O)N1</smiles>

(R)-SQ 32926

(antihypertensive)<smiles>CCOC(=O)C1=C(C)NC(=S)N[C@H]1c1cccc(O)c1</smiles>

(S)-Monastrol<smiles>CC1=C(C(=O)c2ccccc2)C(c2cccc(O)c2)NC(=S)N1C</smiles>

(R)-Mon97

(anticancer agents)

Figure 1. Representative DHPMs with biological activity.

Recently, several examples of enantioselective Biginelli reactions in which asymmetric catalysis with either chiral organometallic complexes ${ }^{7}$ or chiral organocatalysts ${ }^{8}$ is employed, have been described. Of particular relevance to the present report are the advances in highly enantioselective Mannich reactions catalyzed by L-proline. ${ }^{9}$ Indeed, alternative chiral amines have proved efficient as organocatalysts in several asymmetric transformations. ${ }^{10}$ By contrast, L-proline and its corresponding hydrochloride have been used in the Biginelli reaction, but the isolated DHPMs were found to be racemic. ${ }^{11}$

In this context, our research group has developed several novel derivatives of $(1 S, 4 S)-2,5-$ diazabicyclo[2.2.1]heptane as chiral ligands in asymmetric Diels-Alder reactions and in the 
enantioselective diethylzinc addition to benzaldehyde, and as chiral Brønsted acids in $\alpha$-amination of ethyl $\alpha$-cyano- $\alpha$-phenylacetate. ${ }^{10 \mathrm{~d}, 12}$ The present report describes the application of three $(1 S, 4 S)$ 2,5-diazabicyclo[2.2.1]heptane derivatives as chiral organocatalysts in the Biginelli reaction (Figure 2).

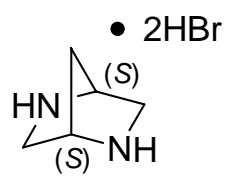

1

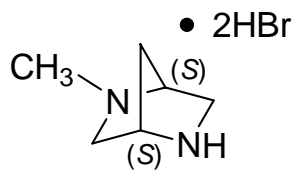

2

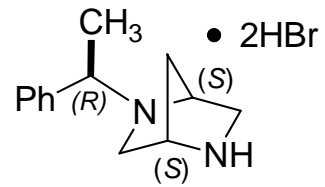

3

Figure 2. (1S,4S)-2,5-Diazabicyclo[2.2.1]heptane $\mathbf{1}$ and derivatives $\mathbf{2}$ and $\mathbf{3}$.

\section{Results and Discussion}

Initially, the potential activities of chiral diamines 1 and $2(10 \mathrm{~mol} \%$, solvent methanol) as organocatalyst in the Biginelli reaction were examined (Table 1). The progress of the reaction between various aldehydes, ethyl acetoacetate and urea was monitored by TLC $\left(\mathrm{CH}_{2} \mathrm{Cl}_{2}-\mathrm{MeOH}\right.$ 95:5). The expected DHPMs were obtained with $\mathbf{1}$ in good yields and moderate enantiomeric purity as established by chiral HPLC (Table 1, entries 1-3). Similar observations were registered with organocatalyst 2 (Table 1, entries 4-6).

Table 1. Use of chiral organocatalysts 1 and 2 in the Biginelli reaction of several aromatic aldehydes with ethyl acetoacetate and urea
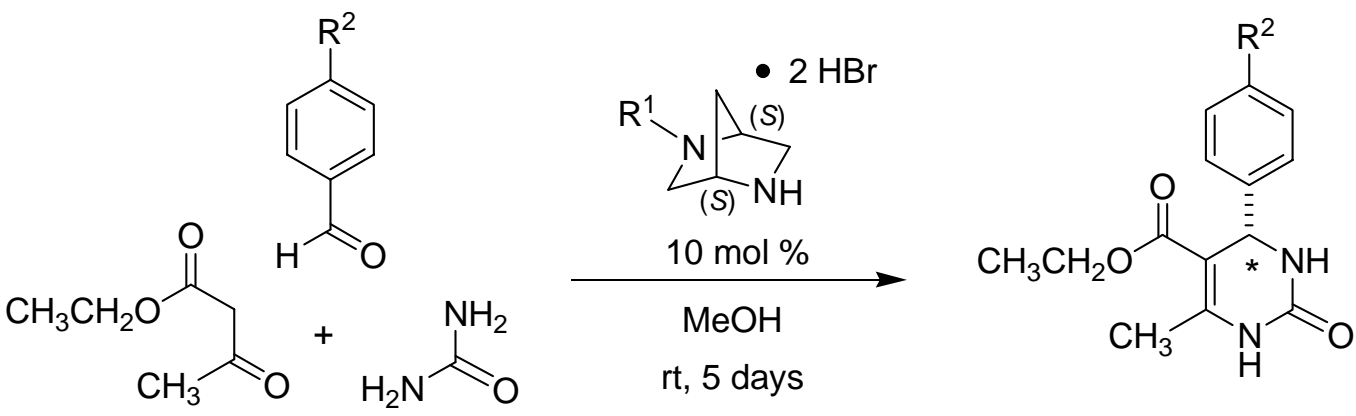

\begin{tabular}{|c|c|c|c|c|c|}
\hline Entry & $\mathrm{R}^{1}$ & $\mathrm{R}^{2}$ & Product & Yield (\%) & $\operatorname{er}(R: S)[\mathrm{ee}]^{\mathrm{a}}$ \\
\hline 1 & $\mathrm{H}$ & $\mathrm{H}$ & 40 & 69 & $37: 63[26]$ \\
\hline 2 & $\mathrm{H}$ & $\mathrm{OCH}_{3}$ & $4 c$ & 61 & $32: 68$ [36] \\
\hline 3 & $\mathrm{H}$ & $\mathrm{Br}$ & $4 k$ & 34 & $41: 59$ [18] \\
\hline 4 & $\mathrm{CH}_{3}$ & $\mathrm{H}$ & 40 & 48 & $36: 64$ [28] \\
\hline 5 & $\mathrm{CH}_{3}$ & $\mathrm{OCH}_{3}$ & $4 c$ & 62 & $31: 69$ [37] \\
\hline 6 & $\mathrm{CH}_{3}$ & $\mathrm{Br}$ & $4 \mathbf{k}$ & 32 & $41: 59[18]$ \\
\hline
\end{tabular}

${ }^{\mathrm{a}}$ Determined by chiral HPLC (see Experimental Section). 
On the other hand, when organocatalyst 3 was used under the same reaction conditions, the corresponding DHPM was obtained in 56\% yield and 32\% ee (Table 2, entry 1). Motivated by this promising result (cf. entries 1 and 4 in Table 1), we deemed it important to further explore this chiral salt as organocatalyst. For instance, the concentration of $\mathbf{3}$ was increased to one half equivalent (50 mol\%); nevertheless, the enantioselectivity did not increase (Table 2, entry 2 ). In other experiment, the methanol solvent was replaced by an isopropanol-methanol 6:1 mixture to find that the yield of product increased substantially to $88 \%$, although the enantiomeric excess remained essentially unchanged, $34 \%$ ee (Table 2 , entry 3 ).

Table 2. Use of organocatalyst 3 under various solvent and catalyst concentration conditions
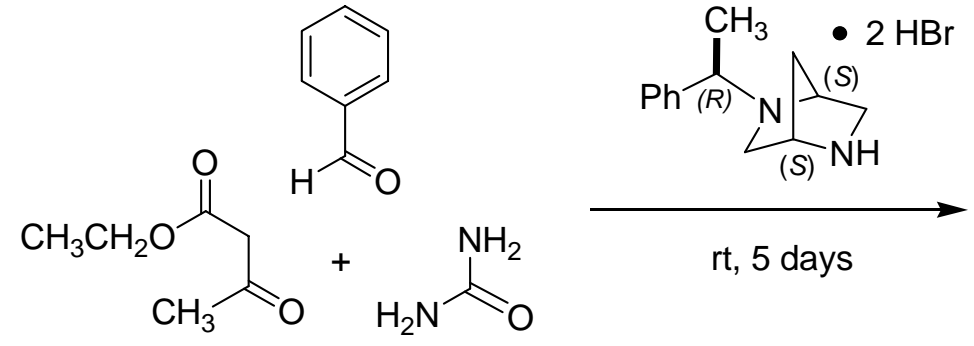

rt, 5 days

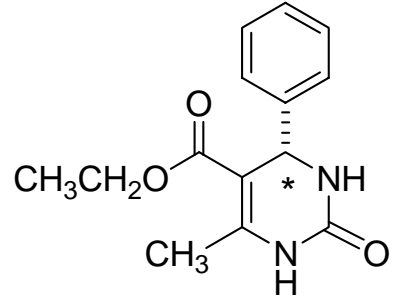

40

\begin{tabular}{ccccc}
\hline Entry & Cat.* $^{(\mathrm{mol} \%)}$ & Solvent & Yield $(\%)$ & er $(R: S)[\mathrm{ee}]^{\mathrm{a}}$ \\
\hline 1 & 10 & $\mathrm{MeOH}$ & 56 & $34: 66[32]$ \\
2 & 50 & $\mathrm{MeOH}$ & 68 & $34: 66[31]$ \\
3 & 10 & $i-\mathrm{PrOH}-\mathrm{MeOH}(6: 1 \mathrm{v} / \mathrm{v})$ & 88 & $33: 67[34]$ \\
\hline
\end{tabular}

${ }^{\mathrm{a}}$ Determined by chiral HPLC (see Experimental Section).

Additional DHPMs were then prepared following the reaction conditions that provided the highest yield of the desired product, $10 \mathrm{~mol} \%$ of catalyst 3, at room temperature during 5 days, and employing different aldehydes. The results are collected in Table 3, finding that electron-donating substituents such as $p$-methoxy and $m$-hydroxy afforded the expected DHPM in good yield and enantioselectivities as high as 46\% (entries 1-4). Interestingly, lower enantioselectivity was observed when the same substituents occupy the meta position or para position (entries 7 and 8 in Table 3). By contrast, moderate electron-withdrawing groups such as chlorine and bromine lead to lower enantiomeric excess, 26\% and 28\% respectively (entries 10 and 11 in Table 3), whereas the strongly electron-withdrawing nitro substituent afforded racemic DHPM (entries 12 and 13 in Table 3 ). Finally, use of methyl acetoacetate instead of ethyl acetoacetate gave similar results (entry 3 in Table 2 and entry 14 in Table 3 ). 
Table 3. Use of organocatalyst 3 in the Biginelli reaction and effect of substitution at the aromatic ring in the aldehyde substrate
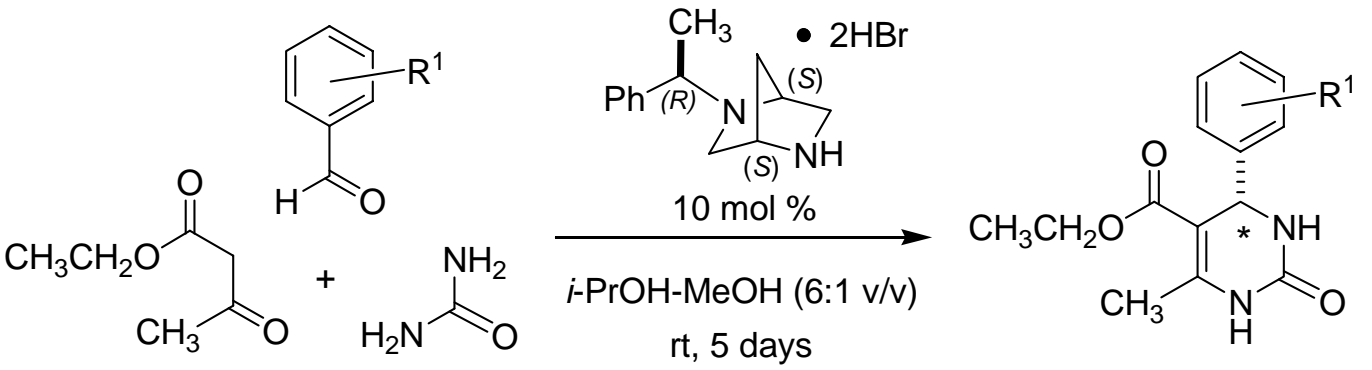

\begin{tabular}{ccccc}
\hline Entry & $\mathrm{R}^{1}$ & Product & Yield (\%) & er $(R: S)[\text { ee }]^{\mathrm{a}}$ \\
\hline 1 & $3,4-\left(\mathrm{OCH}_{3}\right)_{2}$ & $\mathbf{4 a}$ & 71 & $27: 73[46]$ \\
2 & $3-\mathrm{OH}$ & $\mathbf{4 b}$ & 81 & $29: 71[42]$ \\
3 & $4-\mathrm{OCH}_{3}$ & $\mathbf{4 c}$ & 80 & $30: 70[40]$ \\
4 & $3,4-\mathrm{OCH}_{2} \mathrm{O}$ & $\mathbf{4 d}$ & 75 & $30: 70[40]$ \\
5 & $4-\mathrm{CH}_{3}$ & $\mathbf{4 e}$ & 88 & $31: 69[38]$ \\
6 & $4-\mathrm{C}_{6} \mathrm{H}_{5}$ & $\mathbf{4 f}$ & 83 & $32: 68[36]$ \\
7 & $3-\mathrm{OCH}_{3}$ & $\mathbf{4 g}$ & 90 & $34: 66[32]$ \\
8 & $4-\mathrm{OH}$ & $\mathbf{4 h}$ & 75 & $35: 65[30]$ \\
9 & $4-\mathrm{N}$ & $\mathbf{4 i}$ & 27 & $37: 63[26]$ \\
10 & $4-\mathrm{Cl}_{2}$ & $\mathbf{4 j}$ & 79 & $37: 63[26]$ \\
11 & $4-\mathrm{Br}_{2}$ & $\mathbf{4 k}$ & 77 & $36: 64[28]$ \\
12 & $4-\mathrm{NO}_{2}$ & $\mathbf{4 l}$ & 60 & $50: 50[0]$ \\
13 & $3-\mathrm{NO}_{2}$ & $\mathbf{4 m}$ & 55 & $50: 50[0]$ \\
14 & $\mathrm{H}^{\mathrm{b}}$ & $\mathbf{4 n}$ & 94 & $32: 68[36]$ \\
\hline
\end{tabular}

${ }^{a}$ Determined by chiral HPLC. ${ }^{b}$ Methyl acetoacetate was used instead of ethyl acetoacetate.

Seeking to reduce the reaction time in the Biginelli cyclocondensation catalyzed by $(1 S, 4 S)$-2methyl-2,5-diazabicyclo[2.2.1] heptane $2 \mathrm{HBr} 2$, the effect of microwave irradiation (MW) was explored. ${ }^{13,14}$ An exploratory experiment employing 6-7 $\mathrm{W}$ and $45{ }^{\circ} \mathrm{C}$ during $8 \mathrm{~h}$ provided the corresponding product in $42 \%$ yield and $27 \%$ ee (Scheme 2 ). Unfortunately, neither the chemical yield nor the enantiomeric excess could be improved at other temperatures. 


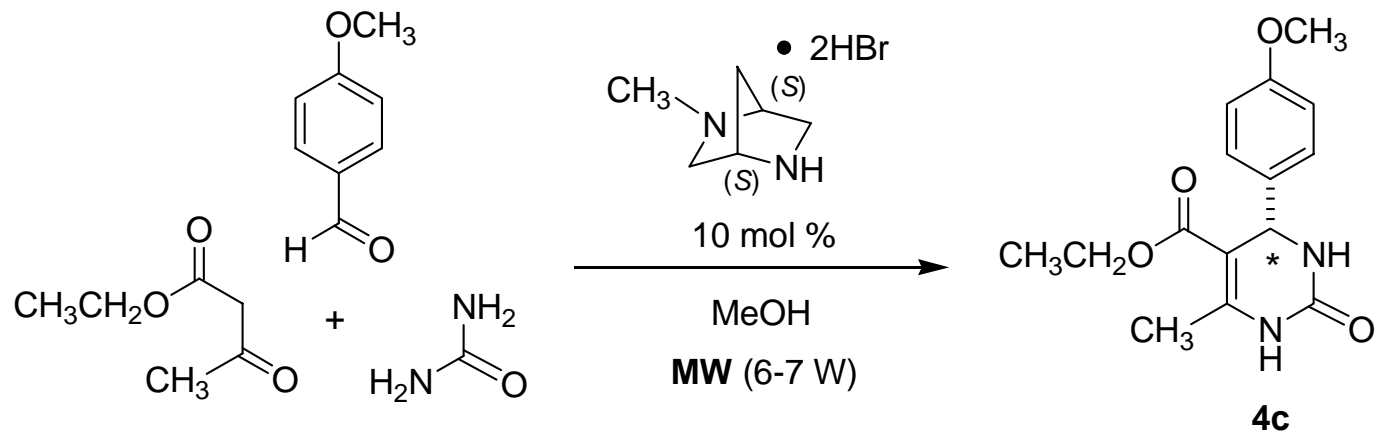

\section{Scheme 2}

Based on mechanistic considerations advanced mainly by List and Kappe ${ }^{15}$ we would consider that the amino catalytic mechanism advanced in Scheme 3 is plausible to interpret the enantioinduction observed in the present reactions.

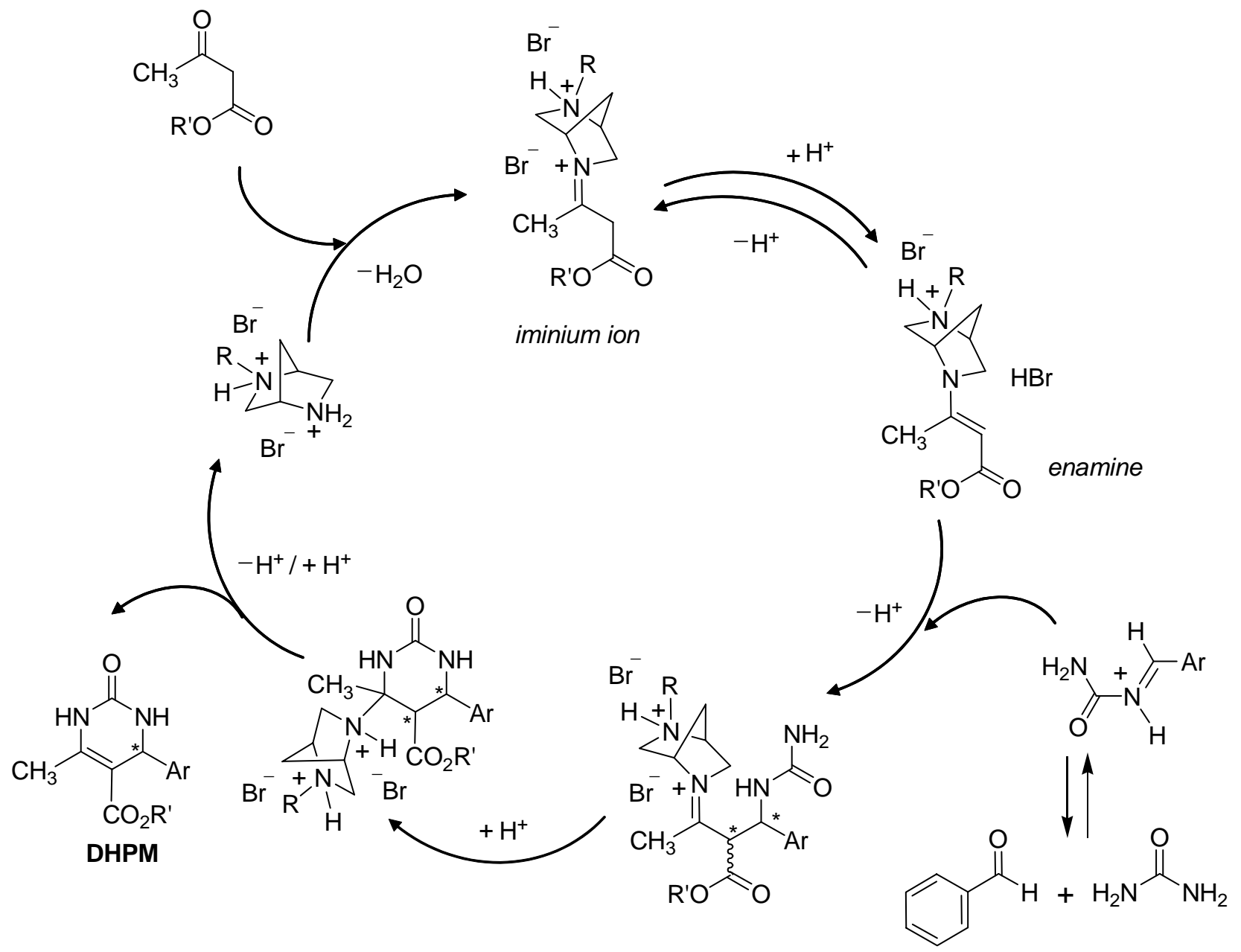

\section{Scheme 3}


In summary, (1S,4S)-2,5-diazadicyclo[2.2.1]heptane derivatives 1-3 catalyze the enantioselective Biginelli cyclocondensation of methyl and ethyl acetoacetate with aromatic aldehydes and urea. The excellent yields and significant enantioselectivities observed in these reactions show that the chiral diamines employed in the present work are promising organocatalysts in asymmetric synthesis.

\section{Experimental Section}

General Procedures. Optical rotations were measured in a Perkin-Elmer 241 polarimeter. NMR spectra were recorded in Bruker Advance 300 (300 MHz) spectrometer. Microwave irradiation was achieved in a singlemode Discover (CEM Corporation) reactor provided with BenchMate and CoolMate accessories. HPLC analyses were carried out with a Waters 600 E equipment fitted with UV/Visible Waters 2487 detector $230 \mathrm{~nm}$ (wavelength) and Chirobiotic T (Astec, Whippany, USA) $(0.46 \times 25 \mathrm{~cm})$ column, employing acetonitrile-water (70:30) mobile phase, and $1 \mathrm{~mL} / \mathrm{min}$ flow.

All Biginelli products obtained in this work (4a-o) were known. ${ }^{16}$ The absolute configuration of the major enantiomer in DHPMs $\mathbf{4 b}, \mathbf{4} \mathbf{k}$ and $\mathbf{4 0}$ was assigned as $(S)$ by comparison with the reported optical rotations for the $(R)$ enantiomers. ${ }^{7 \mathrm{c}}$ The similarity in the sign of the optical rotation for the rest of the DHPMs prepared in this work (dextrorotatory) as well as the similarity in chiral HPLC retention times led to the tentative assignment of the $(\mathrm{S})$ configuration in the major enantiomer.

\section{General procedure for the organocatalytic asymmetric Biginelli reaction}

Organocatalyst $3(18 \mathrm{mg}, 0.05 \mathrm{mmol})$ is dissolved in $0.5 \mathrm{~mL}$ of methanol and $3 \mathrm{~mL}$ of isopropanol before the addition of urea $(0.6 \mathrm{mmol})$, aldehyde $(0.5 \mathrm{mmol})$ and $\beta$-acetoester $(0.5 \mathrm{mmol})$. The resulting mixture is stirred at ambient temperature for 5 days (some of the product starts to precipitate after 12-48 h) following the reaction course by TLC $\left(\mathrm{CH}_{2} \mathrm{Cl}_{2}-\mathrm{MeOH}\right.$ 95:5). The solvent is removed under reduced pressure, and then cold water $(5 \mathrm{~mL})$ is added to the residue to induce precipitation of the product, which is filtered and washed with water to remove the catalyst as well as unreacted urea, and then with hexane. The product is dried $(48 \mathrm{~h})$ at $40{ }^{\circ} \mathrm{C}$ under vacuum.

Scalemic-(+)-Ethyl 4-(3,4-dimethoxyphenyl)-6-methyl-2-oxo-1,2,3,4-tetrahydropyrimidine-5carboxylate (enriched in the $S$ enantiomer) (4a). Yield $71 \% .[\alpha]_{\mathrm{D}}{ }^{20}=+20.0(c 0.5, \mathrm{MeOH})(46 \%$ ee). ${ }^{1} \mathrm{H}$ NMR (DMSO- $\left.d_{6}, 300 \mathrm{MHz}\right): \delta=1.11\left(\mathrm{t},{ }^{3} \mathrm{~J}_{\mathrm{H}, \mathrm{H}}=7 \mathrm{~Hz}, 3 \mathrm{H}, \mathrm{OCH}_{2} \mathrm{CH}_{3}\right), 2.24\left(\mathrm{~s}, 3 \mathrm{H}, \mathrm{CCH}_{3}\right)$, $3.71\left(\mathrm{~s}, 6 \mathrm{H}, \mathrm{ArOCH}_{3}\right), 3.99\left(\mathrm{q},{ }^{3} J_{\mathrm{H}, \mathrm{H}}=7 \mathrm{~Hz}, 2 \mathrm{H}, \mathrm{OCH}_{2} \mathrm{CH}_{3}\right), 5.09\left(\mathrm{~d},{ }^{3} J_{\mathrm{H}, \mathrm{H}}=2.6 \mathrm{~Hz}, 1 \mathrm{H}, \mathrm{CCHNH}\right)$, $6.72\left(\mathrm{~d},{ }^{3} J_{\mathrm{H}, \mathrm{H}}=8.2 \mathrm{~Hz}, 1 \mathrm{H}, \mathrm{CHAr}\right), 6.84-6.90(\mathrm{~m}, 2 \mathrm{H}, \mathrm{CHAr}), 7.69(\mathrm{~s}, 1 \mathrm{H}, \mathrm{NH}), 9.16(\mathrm{~s}, 1 \mathrm{H}, \mathrm{NH})$. ${ }^{13} \mathrm{C}$ NMR (DMSO- $\left.d_{6}, 75.5 \mathrm{MHz}\right): \delta=15.0,18.6,54.3,56.2,56.3,60.0,100.2,111.2,112.5$ 118.7, 138.2, 148.9, 149.0, 149.3, 153.1, 166.3. Enantiomeric ratio: $27: 73$, determined by HPLC: $\mathrm{t}_{\mathrm{r}}=3.40$ $\min$ (minor, $R$ enantiomer); $\mathrm{t}_{\mathrm{r}}=6.02 \mathrm{~min}$ (major, $S$ enantiomer).

Scalemic-(+)-Ethyl 4-(3-hydroxyphenyl)-6-methyl-2-oxo-1,2,3,4-tetrahydropyrimidine-5carboxylate (enriched in the $S$ enantiomer) (4b). Yield $81 \%$. $[\alpha]_{\mathrm{D}}{ }^{20}=+26.0(c 0.5, \mathrm{MeOH})(42 \%$ ee) $\left[\right.$ Lit. $^{7 \mathrm{c}}[\alpha]_{\mathrm{D}}{ }^{20}=-59.0(c 0.5, \mathrm{MeOH}), 91 \%$ ee for the $R$ enantiomer]. ${ }^{1} \mathrm{H}$ NMR (DMSO- $d_{6}, 300$ $\mathrm{MHz}): \delta=1.11\left(\mathrm{t},{ }^{3} J_{\mathrm{H}, \mathrm{H}}=7 \mathrm{~Hz}, 3 \mathrm{H}, \mathrm{OCH}_{2} \mathrm{CH}_{3}\right), 2.23\left(\mathrm{~s}, 3 \mathrm{H}, \mathrm{CCH}_{3}\right), 3.99\left(\mathrm{q},{ }^{3} J_{\mathrm{H}, \mathrm{H}}=7 \mathrm{~Hz}, 2 \mathrm{H}\right.$, 
$\left.\mathrm{OCH}_{2} \mathrm{CH}_{3}\right), 5.05\left(\mathrm{~d},{ }^{3} J_{\mathrm{H}, \mathrm{H}}=2.8 \mathrm{~Hz}, 1 \mathrm{H}, \mathrm{CCHNH}\right), 6.60-6.68(\mathrm{~m}, 3 \mathrm{H}, \mathrm{CHAr}), 7.09\left(\mathrm{t},{ }^{3} J_{\mathrm{H}, \mathrm{H}}=8 \mathrm{~Hz}\right.$, 1H, CHAr), 7.69 (s, 1H, NH), $9.17(\mathrm{~s}, 1 \mathrm{H}, \mathrm{NH}), 9.37$ (s, 1H, OH). ${ }^{13} \mathrm{C}$ NMR (DMSO-d, 75.5 MHz): $\delta=14.9,18.6,54.6,60.0,100.2,113.9,115.0,117.7,130.1,147.1,149.0,153.0,158.2$, 166.2. Enantiomeric ratio: $29: 71, \mathrm{t}_{\mathrm{r}}=3.30 \mathrm{~min}$ (minor, $R$ enantiomer), $\mathrm{t}_{\mathrm{r}}=4.78$ min (major, $S$ enantiomer).

Scalemic-(+)-Ethyl 4-(4-methoxyphenyl)-6-methyl-2-oxo-1,2,3,4-tetrahydropyrimidine-5-carboxylate (enriched in the $S$ enantiomer) (4c). Yield 80\%. $[\alpha]_{\mathrm{D}}{ }^{20}=+17.4$ (c $\left.0.5, \mathrm{MeOH}\right)\left(40 \%\right.$ ee). ${ }^{1} \mathrm{H}$ NMR $\left(\mathrm{DMSO}_{6}, 300 \mathrm{MHz}\right): \delta=1.10\left(\mathrm{t},{ }^{3} J_{\mathrm{H}, \mathrm{H}}=7.08 \mathrm{~Hz}, 3 \mathrm{H}, \mathrm{OCH}_{2} \mathrm{CH}_{3}\right), 2.23\left(\mathrm{~s}, 3 \mathrm{H}, \mathrm{CCH}_{3}\right), 3.71(\mathrm{~s}$, $\left.3 \mathrm{H}, \mathrm{ArOCH}_{3}\right), 3.97\left(\mathrm{q},{ }^{3} J_{\mathrm{H}, \mathrm{H}}=7.07 \mathrm{~Hz}, 2 \mathrm{H}, \mathrm{OCH}_{2} \mathrm{CH}_{3}\right), 5.09\left(\mathrm{~d},{ }^{3} J_{\mathrm{H}, \mathrm{H}}=2.9 \mathrm{~Hz}, 1 \mathrm{H}, \mathrm{CCHNH}\right), 6.87$ $\left(\mathrm{d},{ }^{3} J_{\mathrm{H}, \mathrm{H}}=8.5 \mathrm{~Hz}, 2 \mathrm{H}, \mathrm{CHAr}\right), 7.14\left(\mathrm{~d},{ }^{3} J_{\mathrm{H}, \mathrm{H}}=8.5 \mathrm{~Hz}, 2 \mathrm{H}, \mathrm{CHAr}\right), 7.66(\mathrm{~s}, 1 \mathrm{H}, \mathrm{NH}), 9.15(\mathrm{~s}, 1 \mathrm{H}$, $\mathrm{NH}) .{ }^{13} \mathrm{C}$ NMR (DMSO- $\left.d_{6}, 75.5 \mathrm{MHz}\right): \delta=14.9,18.6,54.2,55.9,60.0,100.4,114.5,128.2,137.9$, 148.9, 153.0, 159.3, 166.2. Enantiomeric ratio: $30: 70, \mathrm{t}_{\mathrm{r}}=3.20 \mathrm{~min}$ (minor, $R$ enantiomer), $\mathrm{t}_{\mathrm{r}}=6.18$ min (major, $S$ enantiomer).

Scalemic-(+)-Ethyl 4-(benzo[d][1,3]dioxol-5-yl)-6-methyl-2-oxo-1,2,3,4-tetrahydro-pyrimidine5-carboxylate (enriched in the $S$ enantiomer) 4d. Yield $75 \%$. $[\alpha]_{\mathrm{D}}{ }^{20}=+15.8(c 0.5, \mathrm{MeOH})$ (40\% ee). ${ }^{1} \mathrm{H}$ NMR (DMSO- $\left.d_{6}, 300 \mathrm{MHz}\right): \delta=1.10\left(\mathrm{t},{ }^{3} J_{\mathrm{H}, \mathrm{H}}=7.0 \mathrm{~Hz}, 3 \mathrm{H}, \mathrm{OCH}_{2} \mathrm{CH}_{3}\right), 2.24(\mathrm{~s}, 3 \mathrm{H}$, $\left.\mathrm{CCH}_{3}\right), 3.98\left(\mathrm{q},{ }^{3} J_{\mathrm{H}, \mathrm{H}}=6.8 \mathrm{~Hz}, 2 \mathrm{H}, \mathrm{OCH}_{2} \mathrm{CH}_{3}\right), 5.06(\mathrm{~s}, 1 \mathrm{H}, \mathrm{CCHNH}), 5.97\left(\mathrm{~s}, 2 \mathrm{H}, \mathrm{OCH}_{2} \mathrm{O}\right), 6.67-$ $6.85(\mathrm{~m}, 3 \mathrm{H}, \mathrm{CHAr}), 7.67(\mathrm{~s}, 1 \mathrm{H}, \mathrm{NH}), 9.16(\mathrm{~s}, 1 \mathrm{H}, \mathrm{NH}) .{ }^{13} \mathrm{C} \mathrm{NMR}\left(\mathrm{DMSO}-d_{6}, 75.5 \mathrm{MHz}\right): \delta=$ 14.9, 18.6, 54.5, 60.0, 100.1, 101.8, 107.5, 108.9, 120.2, 139.7, 147.2, 148.1, 149.2, 152.9, 166.2. Enantiomeric ratio: $30: 70, \mathrm{t}_{\mathrm{r}}=3.22 \mathrm{~min}$ (minor, $R$ enantiomer), $\mathrm{t}_{\mathrm{r}}=4.65 \mathrm{~min}$ (major, $S$ enantiomer).

Scalemic-(+)-Ethyl 6-methyl-2-oxo-4-(4-methylphenyl)-1,2,3,4-tetrahydropyrimidine-5-carboxylate (enriched in the $\boldsymbol{S}$ enantiomer) (4e). Yield $88 \% .[\alpha]_{\mathrm{D}}{ }^{20}=+19.4$ (c 0.5, MeOH) $\left(38 \%\right.$ ee). ${ }^{1} \mathrm{H}$ NMR $\left(\mathrm{DMSO}-d_{6}, 300 \mathrm{MHz}\right): \delta=1.10\left(\mathrm{t},{ }^{3} J_{\mathrm{H}, \mathrm{H}}=7.0 \mathrm{~Hz}, 3 \mathrm{H}, \mathrm{OCH}_{2} \mathrm{CH}_{3}\right), 2.23\left(\mathrm{~s}, 3 \mathrm{H}, \mathrm{CCH}_{3}\right), 2.25(\mathrm{~s}, 3 \mathrm{H}$, $\left.\mathrm{ArCH}_{3}\right), 3.97\left(\mathrm{q},{ }^{3} J_{\mathrm{H}, \mathrm{H}}=7.0 \mathrm{~Hz}, 2 \mathrm{H}, \mathrm{OCH}_{2} \mathrm{CH}_{3}\right), 5.10\left(\mathrm{~d},{ }^{3} J_{\mathrm{H}, \mathrm{H}}=2.5 \mathrm{~Hz}, 1 \mathrm{H}, \mathrm{CCHNH}\right), 7.11(\mathrm{~s}, 4 \mathrm{H}$, CHAr), 7.69 (s, 1H, NH), $9.16(\mathrm{~s}, 1 \mathrm{H}, \mathrm{NH}) .{ }^{13} \mathrm{C}$ NMR (DMSO-d 6 , $\left.75.5 \mathrm{MHz}\right): \delta=14.9,18.6,21.5$, $54.4,60.0,100.2,127.0,129.7,137.2,142.8,149.0,153.0,166.2$. Enantiomeric ratio: 31:69, $\mathrm{t}_{\mathrm{r}}=$ $3.10 \mathrm{~min}$ (minor, $R$ enantiomer), $\mathrm{t}_{\mathrm{r}}=5.22 \mathrm{~min}$ (major, $S$ enantiomer).

Scalemic-(+)-Ethyl 6-methyl-2-oxo-4-(biphenyl-4-yl)-1,2,3,4-tetrahydropyrimidine-5-carboxylate (enriched in the $S$ enantiomer) (4f). Yield 83\%. $[\alpha]_{\mathrm{D}}{ }^{20}=+31.0\left(c \quad 0.5, \mathrm{CH}_{2} \mathrm{Cl}_{2}-\mathrm{MeOH} 1: 1\right)(36 \%$ ee). ${ }^{1} \mathrm{H}$ NMR (DMSO-d $\left.6,300 \mathrm{MHz}\right): \delta=1.13\left(\mathrm{t},{ }^{3} J_{\mathrm{H}, \mathrm{H}}=7.0 \mathrm{~Hz}, 3 \mathrm{H}, \mathrm{OCH}_{2} \mathrm{CH}_{3}\right), 2.26(\mathrm{~s}, 3 \mathrm{H}$, $\left.\mathrm{CCH}_{3}\right), 4.0\left(\mathrm{q},{ }^{3} J_{\mathrm{H}, \mathrm{H}}=7.1 \mathrm{~Hz}, 2 \mathrm{H}, \mathrm{OCH}_{2} \mathrm{CH}_{3}\right), 5.19\left(\mathrm{~d},{ }^{3} J_{\mathrm{H}, \mathrm{H}}=3.0 \mathrm{~Hz}, 1 \mathrm{H}, \mathrm{CCHNH}\right), 7.31-7.37(\mathrm{~m}$, $3 \mathrm{H}, \mathrm{CHAr}), 7.45$ (t, $\left.{ }^{3} J_{\mathrm{H}, \mathrm{H}}=7.5 \mathrm{~Hz}, 2 \mathrm{H}\right), 7.61-7.65$ (m, 4H, CHAr), 7.78 (s, 1H, NH), 9.23 (s, 1H, $\mathrm{NH}) .{ }^{13} \mathrm{C}$ NMR (DMSO-d $\left.6,75.5 \mathrm{MHz}\right): \delta=15.0,18.7,54.5,60.1,100.0,127.5,127.6,127.7$, $128.2,129.8,140.1,140.7,144.8,149.3,153.0,166.2$. Enantiomeric ratio: $32: 68, \mathrm{t}_{\mathrm{r}}=2.95 \mathrm{~min}$ (minor, $R$ enantiomer), $\mathrm{t}_{\mathrm{r}}=5.40$ min (major, $S$ enantiomer).

Scalemic-(+)-Ethyl 4-(3-methoxyphenyl)-6-methyl-2-oxo-1,2,3,4-tetrahydropyrimidine-5-carboxylate (enriched in the $S$ enantiomer) (4g). Yield 90\%. $[\alpha]_{\mathrm{D}}{ }^{20}=+19.0$ (c 0.2, $\left.\mathrm{CH}_{2} \mathrm{Cl}_{2}-\mathrm{EtOH} 7: 3\right)(32 \%$ ee). ${ }^{1} \mathrm{H}$ NMR (DMSO-d, $\left.300 \mathrm{MHz}\right): \delta=1.10\left(\mathrm{t},{ }^{3} J_{\mathrm{H}, \mathrm{H}}=7 \mathrm{~Hz}, 3 \mathrm{H}, \mathrm{OCH}_{2} \mathrm{CH}_{3}\right), 2.24\left(\mathrm{~s}, 3 \mathrm{H}, \mathrm{CCH}_{3}\right), 3.72$ $\left(\mathrm{s}, 3 \mathrm{H}, \mathrm{ArOCH}_{3}\right), 3.99\left(\mathrm{q},{ }^{3} J_{\mathrm{H}, \mathrm{H}}=7 \mathrm{~Hz}, 2 \mathrm{H}, \mathrm{OCH}_{2} \mathrm{CH}_{3}\right), 5.11\left(\mathrm{~d},{ }^{3} J_{\mathrm{H}, \mathrm{H}}=2.2 \mathrm{~Hz}, 1 \mathrm{H}, \mathrm{CCHNH}\right)$, 6.77-6.83 (m, 3H, CHAr), $7.24\left(\mathrm{t},{ }^{3} J_{\mathrm{H}, \mathrm{H}}=7.8 \mathrm{~Hz}, 1 \mathrm{H}, \mathrm{CHAr}\right), 7.73(\mathrm{~s}, 1 \mathrm{H}, \mathrm{NH}), 9.19(\mathrm{~s}, 1 \mathrm{H}, \mathrm{NH})$. ${ }^{13} \mathrm{C}$ NMR (DMSO- $\left.d_{6}, 75.5 \mathrm{MHz}\right): \delta=15.0,18.6,54.6,55.8,60.1,100.0,113.0,113.2,119.1,130.4$, 
147.2, 149.3, 153.1, 160.0, 166.2. Enantiomeric ratio: $34: 66, \mathrm{t}_{\mathrm{r}}=3.17 \min$ (minor, $R$ enantiomer), $\mathrm{t}_{\mathrm{r}}$ $=4.53$ min (major, $S$ enantiomer).

Scalemic-(+)-Ethyl 4-(4-hydroxyphenyl)-6-methyl-2-oxo-1,2,3,4-tetrahydropyrimidine-5-carboxylate (enriched in the $\boldsymbol{S}$ enantiomer) (4h). Yield 75\%. $[\alpha]_{\mathrm{D}}{ }^{20}=+17.7$ (c $\left.0.5, \mathrm{MeOH}\right)\left(30 \%\right.$ ee). ${ }^{1} \mathrm{H}$ NMR $\left(\mathrm{DMSO}-d_{6}, 300 \mathrm{MHz}\right): \delta=1.09\left(\mathrm{t},{ }^{3} J_{\mathrm{H}, \mathrm{H}}=7 \mathrm{~Hz}, 3 \mathrm{H}, \mathrm{OCH}_{2} \mathrm{CH}_{3}\right), 2.23\left(\mathrm{~s}, 3 \mathrm{H}, \mathrm{CCH}_{3}\right), 3.97\left(\mathrm{q},{ }^{3} J_{\mathrm{H}, \mathrm{H}}\right.$ $\left.=7 \mathrm{~Hz}, 2 \mathrm{H}, \mathrm{OCH}_{2} \mathrm{CH}_{3}\right), 5.04\left(\mathrm{~d},{ }^{3} J_{\mathrm{H}, \mathrm{H}}=2.2 \mathrm{~Hz}, 1 \mathrm{H}, \mathrm{CCHNH}\right), 6.68\left(\mathrm{~d},{ }^{3} J_{\mathrm{H}, \mathrm{H}}=8.3 \mathrm{~Hz}, 2 \mathrm{H}, \mathrm{CHAr}\right)$, $7.02\left(\mathrm{~d},{ }^{3} J_{\mathrm{H}, \mathrm{H}}=8.3 \mathrm{~Hz}, 2 \mathrm{H}, \mathrm{CHAr}\right), 7.61(\mathrm{~s}, 1 \mathrm{H}, \mathrm{NH}), 9.10(\mathrm{~s}, 1 \mathrm{H}, \mathrm{NH}), 9.32(\mathrm{~s}, 1 \mathrm{H}, \mathrm{OH}) .{ }^{13} \mathrm{C}$ NMR $\left(\mathrm{DMSO}_{6}, 75.5 \mathrm{MHz}\right): \delta=14.9,18.6,54.3,59.9,100.6,115.8,128.2,136.3,148.6,153.0,157.4$, 166.3. Enantiomeric ratio: $35: 65, \mathrm{t}_{\mathrm{r}}=3.33$ min (minor, $R$ enantiomer), $\mathrm{t}_{\mathrm{r}}=6.47$ min (major, $S$ enantiomer).

Scalemic-(+)-Ethyl 4-(4-( $N, N$-dimethylamino)phenyl)-6-methyl-2-oxo-1,2,3,4-tetrahydropyrimidine-5carboxylate (enriched in the $S$ enantiomer) (4i). Yield $27 \%$. $[\alpha]_{\mathrm{D}}{ }^{20}=+14.0(c) .5, \mathrm{CH}_{2} \mathrm{Cl}_{2}-\mathrm{MeOH}$ $1: 1)\left(26 \%\right.$ ee). ${ }^{1} \mathrm{H}$ NMR (DMSO-d, $\left.300 \mathrm{MHz}\right): \delta=1.11\left(\mathrm{t},{ }^{3} J_{\mathrm{H}, \mathrm{H}}=6.8 \mathrm{~Hz}, 3 \mathrm{H}, \mathrm{OCH}_{2} \mathrm{CH}_{3}\right), 2.22(\mathrm{~s}$, $\left.3 \mathrm{H}, \mathrm{CCH}_{3}\right), 2.84\left(\mathrm{~s}, 6 \mathrm{H}, \operatorname{ArN}\left(\mathrm{CH}_{3}\right)_{2}\right), 3.97\left(\mathrm{q},{ }^{3} \mathrm{~J}_{\mathrm{H}, \mathrm{H}}=6.7 \mathrm{~Hz}, 2 \mathrm{H}, \mathrm{OCH}_{2} \mathrm{CH}_{3}\right), 5.02(\mathrm{~s}, 1 \mathrm{H}$, $\mathrm{CCHNH}), 6.65\left(\mathrm{~d},{ }^{3} J_{\mathrm{H}, \mathrm{H}}=7.9 \mathrm{~Hz}, 2 \mathrm{H}, \mathrm{CHAr}\right), 7.03\left(\mathrm{~d},{ }^{3} J_{\mathrm{H}, \mathrm{H}}=7.8 \mathrm{~Hz}, 2 \mathrm{H}, \mathrm{CHAr}\right), 7.59(\mathrm{~s}, 1 \mathrm{H}$, $\mathrm{NH}), 9.10(\mathrm{~s}, 1 \mathrm{H}, \mathrm{NH}) .{ }^{13} \mathrm{C} \mathrm{NMR}\left(\mathrm{DMSO}_{6}, 75.5 \mathrm{MHz}\right): \delta=15.0,18.6,41.0,54.1,59.9,100.7$, $113.1,127.7,133.5,148.4,150.6,153.1,166.3$. Enantiomeric ratio: $37: 63, \mathrm{t}_{\mathrm{r}}=3.47$ min (minor, $R$ enantiomer), $\mathrm{t}_{\mathrm{r}}=7.38 \mathrm{~min}$ (major, $S$ enantiomer).

Scalemic-(+)-Ethyl 4-(4-chlorophenyl)-6-methyl-2-oxo-1,2,3,4-dihydropyrimidine-5-carboxylate (enriched in the $\boldsymbol{S}$ enantiomer) (4j). Yield 79\%. $[\alpha]_{\mathrm{D}}{ }^{20}=+17.1(c 0.5, \mathrm{MeOH})\left(26 \%\right.$ ee). ${ }^{1} \mathrm{H} \mathrm{NMR}$ $\left(\mathrm{DMSO}_{6}, 300 \mathrm{MHz}\right): \delta=1.09\left(\mathrm{t},{ }^{3} J_{\mathrm{H}, \mathrm{H}}=7 \mathrm{~Hz}, 3 \mathrm{H}, \mathrm{OCH}_{2} \mathrm{CH}_{3}\right), 2.25\left(\mathrm{~s}, 3 \mathrm{H}, \mathrm{CCH}_{3}\right), 3.98\left(\mathrm{q},{ }^{3} J_{\mathrm{H}, \mathrm{H}}\right.$ $\left.=7 \mathrm{~Hz}, 2 \mathrm{H}, \mathrm{OCH}_{2} \mathrm{CH}_{3}\right), 5.14\left(\mathrm{~d},{ }^{3} J_{\mathrm{H}, \mathrm{H}}=2.8 \mathrm{~Hz}, 1 \mathrm{H}, \mathrm{CCHNH}\right), 7.25\left(\mathrm{~d},{ }^{3} J_{\mathrm{H}, \mathrm{H}}=8.4 \mathrm{~Hz}, 2 \mathrm{H}, \mathrm{CHAr}\right)$, $7.39\left(\mathrm{~d},{ }^{3} J_{\mathrm{H}, \mathrm{H}}=8.3 \mathrm{~Hz}, 2 \mathrm{H}, \mathrm{CHAr}\right), 7.77(\mathrm{~s}, 1 \mathrm{H}, \mathrm{NH}), 9.24(\mathrm{~s}, 1 \mathrm{H}, \mathrm{NH}) .{ }^{13} \mathrm{C} \mathrm{NMR}\left(\mathrm{DMSO}-d_{6}, 75.5\right.$ $\mathrm{MHz}): \delta=14.9,18.6,54.3,60.1,99.7,129.0,129.2,132.6,144.6,149.6,152.8,166.0$. Enantiomeric ratio: $37: 63, \mathrm{t}_{\mathrm{r}}=3.07 \mathrm{~min}$ (minor, $R$ enantiomer), $\mathrm{t}_{\mathrm{r}}=5.60 \mathrm{~min}$ (major, $S$ enantiomer).

Scalemic-(+)-Ethyl 4-(4-bromophenyl)-6-methyl-2-oxo-1,2,3,4-tetrahydropyrimidine-5-carboxylate (enriched in the $\boldsymbol{S}$ enantiomer) (4k). Yield 77\%. $[\alpha]_{\mathrm{D}}{ }^{20}=+14.6(c 0.5, \mathrm{MeOH})\left(28 \%\right.$ ee) [Lit. $^{7 \mathrm{c}}$ $[\alpha]_{\mathrm{D}}{ }^{20}=-65(c 0.5, \mathrm{MeOH}), 95 \%$ ee for the $R$ enantiomer]. ${ }^{1} \mathrm{H}$ NMR (DMSO- $\left.d_{6}, 300 \mathrm{MHz}\right): \delta=$ $1.09\left(\mathrm{t},{ }^{3} J_{\mathrm{H}, \mathrm{H}}=7.04 \mathrm{~Hz}, 3 \mathrm{H}, \mathrm{OCH}_{2} \mathrm{CH}_{3}\right), 2.24\left(\mathrm{~s}, 3 \mathrm{H}, \mathrm{CCH}_{3}\right), 3.98\left(\mathrm{q},{ }^{3} J_{\mathrm{H}, \mathrm{H}}=7.04 \mathrm{~Hz}, 2 \mathrm{H}\right.$, $\left.\mathrm{OCH}_{2} \mathrm{CH}_{3}\right), 5.12\left(\mathrm{~d},{ }^{3} J_{\mathrm{H}, \mathrm{H}}=3.0 \mathrm{~Hz}, 1 \mathrm{H}, \mathrm{CCHNH}\right), 7.18\left(\mathrm{~d},{ }^{3} J_{\mathrm{H}, \mathrm{H}}=8.3 \mathrm{~Hz}, 2 \mathrm{H}, \mathrm{CHAr}\right), 7.52(\mathrm{~d}$, $\left.{ }^{3} J_{\mathrm{H}, \mathrm{H}}=8.2 \mathrm{~Hz}, 2 \mathrm{H}, \mathrm{CHAr}\right), 7.75(\mathrm{~s}, 1 \mathrm{H}, \mathrm{NH}), 9.22(\mathrm{~s}, 1 \mathrm{H}, \mathrm{NH}) .{ }^{13} \mathrm{C} \mathrm{NMR}\left(\mathrm{DMSO}-d_{6}, 75.5 \mathrm{MHz}\right): \delta$ $=14.9,18.6,54.3,60.1,99.6,121.1,129.4,132.2$, 145.0, 149.6, 152.8, 166.1. Enantiomeric ratio: $36: 64, \mathrm{t}_{\mathrm{r}}=3.08 \mathrm{~min}$ (minor, $R$ enantiomer), $\mathrm{t}_{\mathrm{r}}=5.40 \mathrm{~min}$ (major, $S$ enantiomer).

Ethyl 6-methyl-4-(4-nitrophenyl)-2-oxo-1,2,3,4-tetrahydropyrimidine-5-carboxylate (enriched in the $S$ enantiomer) (4l). Yield 60\% (racemic product). ${ }^{1} \mathrm{H}$ NMR (DMSO- $d_{6}, 300 \mathrm{MHz}$ ): $\delta=1.09$ $\left(\mathrm{t},{ }^{3} J_{\mathrm{H}, \mathrm{H}}=7.0 \mathrm{~Hz}, 3 \mathrm{H}, \mathrm{OCH}_{2} \mathrm{CH}_{3}\right), 2.26\left(\mathrm{~s}, 3 \mathrm{H}, \mathrm{CCH}_{3}\right), 3.98\left(\mathrm{q},{ }^{3} J_{\mathrm{H}, \mathrm{H}}=6.9 \mathrm{~Hz}, 2 \mathrm{H}, \mathrm{OCH}_{2} \mathrm{CH}_{3}\right), 5.26$ $(\mathrm{s}, 1 \mathrm{H}, \mathrm{CCHNH}), 7.50\left(\mathrm{~d},{ }^{3} J_{\mathrm{H}, \mathrm{H}}=7.6 \mathrm{~Hz}, 2 \mathrm{H}, \mathrm{CHAr}\right), 7.90(\mathrm{~s}, 1 \mathrm{H}, \mathrm{NH}), 8.21\left(\mathrm{~d},{ }^{3} J_{\mathrm{H}, \mathrm{H}}=7.7 \mathrm{~Hz}, 2 \mathrm{H}\right.$, CHAr), 9.36 (s, 1H, NH). ${ }^{13} \mathrm{C}$ NMR (DMSO-d 6 , $\left.75.5 \mathrm{MHz}\right): \delta=14.9,18.7,54.5,60.2,99.0,124.7$, 128.5, 147.6, 150.3, 152.6, 152.8, 165.9.

Ethyl 6-methyl-4-(3-nitrophenyl)-2-oxo-1,2,3,4-tetrahydropyrimidine-5-carboxylate (enriched in the $S$ enantiomer) (4m). Yield 55\% (racemic product). ${ }^{1} \mathrm{H}$ NMR (DMSO- $\left.d_{6}, 300 \mathrm{MHz}\right): \delta=$ 
$1.09\left(\mathrm{t},{ }^{3} J_{\mathrm{H}, \mathrm{H}}=7.0 \mathrm{~Hz}, 3 \mathrm{H}, \mathrm{OCH}_{2} \mathrm{CH}_{3}\right), 2.26\left(\mathrm{~s}, 3 \mathrm{H}, \mathrm{CCH}_{3}\right), 3.98\left(\mathrm{q},{ }^{3} J_{\mathrm{H}, \mathrm{H}}=7.0 \mathrm{~Hz}, 2 \mathrm{H}, \mathrm{OCH}_{2} \mathrm{CH}_{3}\right)$, $5.30\left(\mathrm{~d},{ }^{3} J_{\mathrm{H}, \mathrm{H}}=2.5 \mathrm{~Hz}, 1 \mathrm{H}, \mathrm{CCHNH}\right), 7.62-7.71(\mathrm{~m}, 2 \mathrm{H}, \mathrm{CHAr}), 7.90(\mathrm{~s}, 1 \mathrm{H}, \mathrm{NH}), 8.08-8.15(\mathrm{~m}$, $2 \mathrm{H}, \mathrm{CHAr}), 9.37(\mathrm{~s}, 1 \mathrm{H}, \mathrm{NH}) .{ }^{13} \mathrm{C}$ NMR (DMSO-d $\left.6,75.5 \mathrm{MHz}\right): \delta=14.9,18.7,54.4,60.2,99.1$, $121.9,123.2,131.1,133.8,147.8,148.6,150.3,152.6,165.9$.

Scalemic-(+)-Methyl 6-methyl-2-oxo-4-phenyl-1,2,3,4-tetrahydropyrimidine-5-carboxylate (enriched in the $S$ enantiomer) (4n). Yield 94\%. $[\alpha]_{\mathrm{D}}{ }^{20}=+17$ (c 0.5, MeOH) $\left(36 \%\right.$ ee). ${ }^{1} \mathrm{H}$ NMR (DMSO- $d_{6}, 300$ $\mathrm{MHz}): \delta=2.25\left(\mathrm{~s}, 3 \mathrm{H}, \mathrm{CCH}_{3}\right), 3.53\left(\mathrm{~s}, 3 \mathrm{H}, \mathrm{OCH}_{3}\right), 5.15\left(\mathrm{~d},{ }^{3} \mathrm{~J}_{\mathrm{H}, \mathrm{H}}=2.8 \mathrm{~Hz}, 1 \mathrm{H}, \mathrm{CCHNH}\right), 7.22-$ $7.35(\mathrm{~m}, 5 \mathrm{H}, \mathrm{CHAr}), 7.76(\mathrm{~s}, 1 \mathrm{H}, \mathrm{NH}), 9.22(\mathrm{~s}, 1 \mathrm{H}, \mathrm{NH}) .{ }^{13} \mathrm{C}$ NMR (DMSO-d $\left.6,75.5 \mathrm{MHz}\right): \delta=$ 18.7, 51.6, 54.6, 99.8, 127.0, 128.1, 129.3, 145.5, 149.5, 153.0, 166.7. Enantiomeric ratio: 32:68, $\mathrm{t}_{\mathrm{r}}$ $=3.30 \mathrm{~min}$ (minor, $R$ enantiomer), $\mathrm{t}_{\mathrm{r}}=6.08 \mathrm{~min}$. (major, $S$ enantiomer).

Scalemic-(+)-Ethyl 6-methyl-2-oxo-4-phenyl-1,2,3,4-tetrahydropyrimidine-5-carboxylate (enriched in the $S$ enantiomer) (4o). Yield $88 \% .[\alpha]_{\mathrm{D}}{ }^{20}=+14.4(c 0.5, \mathrm{MeOH})\left(32 \%\right.$ ee) $\left[\right.$ Lit. $^{7 \mathrm{c}}[\alpha]_{\mathrm{D}}{ }^{20}=-58(c 0.5$, $\mathrm{MeOH}), 90 \%$ ee for the $R$ enantiomer]. ${ }^{1} \mathrm{H}$ NMR (DMSO- $\left.d_{6}, 300 \mathrm{MHz}\right): \delta=1.08\left(\mathrm{t},{ }^{3} J_{\mathrm{H}, \mathrm{H}}=7.07\right.$ $\left.\mathrm{Hz}, 3 \mathrm{H}, \mathrm{OCH}_{2} \mathrm{CH}_{3}\right), 2.24\left(\mathrm{~s}, 3 \mathrm{H}, \mathrm{CCH}_{3}\right), 3.97\left(\mathrm{q},{ }^{3} J_{\mathrm{H}, \mathrm{H}}=7.07 \mathrm{~Hz}, 2 \mathrm{H}, \mathrm{OCH}_{2} \mathrm{CH}_{3}\right), 5.14\left(\mathrm{~d},{ }^{3} J_{\mathrm{H}, \mathrm{H}}=3\right.$ $\mathrm{Hz}, 1 \mathrm{H}, \mathrm{CCHNH}), 7.22-7.34(\mathrm{~m}, 5 \mathrm{H}, \mathrm{CHAr}), 7.72(\mathrm{~s}, 1 \mathrm{H}, \mathrm{NH}), 9.17(\mathrm{~s}, 1 \mathrm{H}, \mathrm{NH}) .{ }^{13} \mathrm{C} \mathrm{NMR}$ (DMSO- $\left.d_{6}, 75.5 \mathrm{MHz}\right): \delta=14.9,18.6,54.8,60.0,100.1,127.1,128.1,129.2,145.7,149.2,152.9$, 166.2. Enantiomeric ratio: $34: 66, \mathrm{t}_{\mathrm{r}}=3.18$ min (minor, $R$ enantiomer), $\mathrm{t}_{\mathrm{r}}=5.45$ min (major, $S$ enantiomer).

\section{Acknowledgements}

The authors are grateful to CONACyT, México, for financial support via grant No. 45157-Q and to DGAPA-UNAM, via grant No. PAPIIT IN-202701.

\section{References}

1. (a) Kappe, C. O. In Multicomponent Reactions; Zhu, J.; Bienaymé, H., Eds.; Wiley-VCH: Weinheim, Germany, 2005, pp 95-120. (b) Tietze, L. F.; Brasche, G.; Gericke, K. M. In Domino Reactions in Organic Synthesis; Wiley-VCH: Weinheim, Germany, 2006, pp 542-565.

2. (a) Biginelli, P. Gazz. Chim. Ital. 1893, 23, 360. For recent reviews, see: (b) Kappe, C. O. Tetrahedron 1993, 49, 6937. (c) Kappe, C. O. Acc. Chem. Res. 2000, 33, 879.

3. Kappe, C. O. Eur. J. Med. Chem. 2000, 35, 1043.

4. Atwal, K. S.; Swanson, B. N.; Unger, S. E.; Floyd, D. M.; Moreland, S.; Hedberg, A.; O’Reilly, B. C. J. Med. Chem. 1991, 34, 806.

5. (a) Mayer, T. U.; Kapoor, T. M.; Haggarty, S. J.; King, R. W.; Schreiber, S. L.; Mitchison, T. J. Science 1999, 286, 971. (b) Russowsky, D.; Canto, R. F. S.; Sanches, S. A. A.; D’Oca, M. G. M.; de Fátima, Â.; Pilli, R. A.; Kohn, L. K.; Antônio, M. A.; de Carvalho, J. E. Bioorg. Chem. 2006, 34, 173. 
6. Garcia-Saez, I.; DeBonis, S.; Lopez, R.; Trucco, F.; Rousseau, B.; Thuéry, P.; Kozielski, F. J. Biol. Chem. 2007, 282, 9740.

7. (a) Muñoz-Muñiz, O.; Juaristi, E. Arkivoc 2003, (xi), 16. (b) Corrigendum: González-Olvera, R.; Melgar-Fernández, R.; Juaristi, E. Arkivoc 2003, (xi), 227. (c) Huang, Y.; Yang, F.; Zhu, C. J. Am. Chem. Soc. 2005, 127, 16386.

8. (a) Chen, X.-H.; Xu, X.-Y.; Liu, H.; Cun, L.-F.; Gong, L.-Z. J. Am. Chem. Soc. 2006, 128, 14802. (b) Gong, L.-Z.; Chen, X.-H.; Xu, X.-Y. Chem. Eur. J. 2007, 13, 8920.

9. (a) List, B. J. Am. Chem. Soc. 2000, 122, 9336. (b) Cordova, A.; Notz, W.; Zhong, G.; Betancort, J. M.; Barbas III, C. F. J. Am. Chem. Soc. 2002, 124, 1842. (c) List, B.; Pojarliev, P.; Biller, W. T.; Martin, H. J. J. Am. Chem. Soc. 2002, 124, 827. (d) Notz, W.; Watanabe, S.; Chowdari, N. S.; Zhong, G.; Betancort, J. M.; Tanaka, F.; Barbas III, C. F. Adv. Synth. Catal. 2004, 346, 1131. (e) Zhuang, W.; Saaby, S.; Jorgensen, K. A. Angew. Chem. Int. Ed. 2004, 43, 4476. (f) Cobb, A. J. A.; Shaw, D. M.; Ley, S. V. Synlett 2004, 558. (g) Wang, W.; Wang, J.; Li, H. Tetrahedron Lett. 2004, 45, 7243. (h) Enders, D.; Grondal, C.; Vrettou, M.; Raabe, G. Angew. Chem., Int. Ed. 2005, 44, 4079. (i) Westermann, B.; Neuhaus, C. Angew. Chem., Int. Ed. 2005, 44, 4077. (j) Fustero, S.; Jimenez, D.; Sanz-Cervera, J. F.; Sanchez-Rosello, M.; Esteban, E.; Simon-Fuentes, A. Org. Lett. 2005, 7, 3433. (k) Janey, J. M.; Hsiao, Y.; Armstrong III, J. D. J. Org. Chem. 2006, 71, 390. (1) Yang, J. W.; Stadler, M.; List, B. Angew. Chem., Int. Ed. 2007, 46, 609. (m) Guillena, G.; Ramón, D. J.; Yus, M. Tetrahedron: Asymmetry 2007, 18, 693.

10. (a) Berkessel A.; Gröger, H., Eds.; Asymmetric Organocatalysis: From Biomimetic Concepts to Applications in Asymmetric Synthesis; Wiley-VCH: Weinheim, Germany, 2005. (b) Austin, J. F.; MacMillan, D. W. C. J. Am. Chem. Soc. 2002, 124, 1172. (c) Chen, Y. K.; Yoshida, M.; MacMillan, D. W. C. J. Am. Chem. Soc. 2006, 128, 9328. (d) Liu, Y.; Melgar-Fernández, R.; Juaristi, E. J. Org. Chem. 2007, 72, 1522. (e) Lou, S.; Dai, P.; Shaus, S. E. J. Org. Chem. 2007, 72, 9998.

11. (a) Yadav, J. S.; Kumar, S. P.; Kondaji, G.; Rao, R. S.; Nagaiah, K. Chem. Lett. 2004, 33, 1168. (b) Mabry, J.; Ganem, B. Tetrahedron Lett. 2006, 47, 55.

12. Melgar-Fernández, R.; González-Olvera, R.; Olivares-Romero, J. L.; González-López, V.; Romero-Ponce, L.; Ramírez-Zárate, M. R.; Demare, P.; Regla, I.; Juaristi, E. Eur. J. Org. Chem. 2008, 2008, 655.

13. MW experiments were carried out in a "Discover" reactor provided with the BenchMate system, CEM Corporation.

14. For other asymmetric reactions assisted by microwave irradiation, see: (a) Westermann, B.; Neuhaus, C. Angew. Chem. Int. Ed. 2005, 44, 4077. (b) Rodríguez, B.; Bolm, C. J. Org. Chem. 2006, 71, 2888. (c) Mossé, S.; Alexakis, A. Org. Lett. 2006, 8, 3577. (d) Hosseini, M.; Stiasni, N.; Barbieri, V.; Kappe, C. O. J. Org. Chem. 2007, 72, 1417.

15. (a) List, B. Chem. Commun. 2006, 819. (b) List, B. Synlett 2001, 1675. (c) Kappe, C. O. J. Org. Chem. 1997, 62, 7201.

16. (a) Sujatha, K.; Shanmugam, P.; Perumal, P. T.; Muralidharan, D.; Rajendran, M. Bioorg. Med. Chem. Lett. 2006, 16, 4893. (b) Zhang, M.; Li, Y.-Q. Synth. Commun. 2006, 36, 835. (c) Lu, J.; 
Bai, Y. Synthesis 2002, 466. (d) Fu, N.-Y.; Yuan, Y.-F.; Cao, Z.; Wang, S.-W.; Wang, J.-T.; Peppe, C. Tetrahedron 2002, 58, 4801. (e) J. Chem. Soc., Perkin Trans. 1, 2001, 1939. (f) Ranu, B. C.; Hajra, A.; Jana, U. J. Org. Chem. 2000, 65, 6270. 